\title{
La especificación de
}

procesos en las instituciones públicas de educación superior

DOI: $10.22403 /$ UQROOMX/TYP02/09

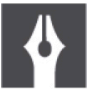

Resumen

\author{
Adalberto Velázquez Méndez* \\ Armando Maldonado Talamantes
}

En este trabajo se presenta una propuesta de especificación de los procesos esenciales (sustantivos) y de apoyo (adjetivos) que se llevan a cabo en las instituciones de educación superior (IES) de carácter público. Cada uno de los procesos es desglosado en macro actividades (subprocesos) correspondientes a una funcionalidad específica en el negocio de las IES. El orden lógico (flujo de trabajo) en que estas funciones son realizadas, las actividaes y acciones comprendidas en ellas y los responsables de realizarlas, así como las relaciones existentes entre los subprocesos y el proceso que integran, conforman un marco de referencia conceptual que las IES pueden difundir entre todos los involucrados (trabajadores del negocio y unidades organizacionales) en las actividades generadoras de valor en la institución, propiciando el entendimiento común y extendiendo esta percepción hasta los sistemas de información (Tו) que soportan el negocio.

\section{Palabras Arquitectura de procesos, especificación de procesos y CLAVE actividades, institución de educación superior, modelo de negocios, servicios de información.}

*Universidad de Quintana Roo / avelazquezm@correo.uqroo.mx

**Instituto Tecnológico Autónomo de México / maldonado@itam.mx 


\section{Antecedentes}

Uno de los modelos que mejor proporcionan una idea general del quehacer de una organización es la arquitectura de procesos (Maldonado, 2003). En este modelo, la organización es desglosada en función de sus procesos fundamentales $y$ de las relaciones existentes entre ellos y con otros procesos de soporte y administración. Este trabajo fue desarrollado con base en la observación de una Institución de Educación Superior (IES) a través de sus procesos fundamentales (Docencia, Investigación y Extensión) y los de soporte (Apoyo Académico, Apoyo Institucional y Administración de Planta Física), presentada en el primer número de la revista Teoría y Praxis.

\section{Metodología}

La arquitectura de procesos comprende el portafolio de funciones core que conducen las actividades en toda IES y conforma la ventaja competitiva de la institución sobre sus competidores. Estos procesos, por su importancia en la generación de valor para la institución, son merecedores de una especificación más detallada (modelado) de las actividades que se realizan en ellos.

¿Cómo se especifican los procesos fundamentales de una IES?, partiendo del modelo de la arquitectura de procesos y retomando los elementos que comprenden la cadena de valor (Porter, 2002), la vista horizontal (Rummler, 1991) y la estructura organizacional de la IES.

I. Se especifica la arquitectura de procesos (Velázquez y Maldonado, 2005)

2. Se identifican las actividades que comprende cada proceso en el modelo de configuración de valor de la IES, asimismo, sus ligas con otras actividades, clientes y proveedores que fueron especificadas al momento de construir el modelo de la vista horizontal de la IES.

3. Las actividades identificadas se agrupan en macro actividades o subprocesos, integrando así, actividades funcionales fragmentadas que son realizadas en diversas unidades organizacionales de la IES y el orden lógico (flujo de trabajo) en que estas funciones son realizadas.

4. Cada uno de los subprocesos es descrito detalladamente, especificando las actividades y acciones comprendidas en ellos, los responsables de 
realizarlas, así como las relaciones existentes entre los subprocesos y el proceso que integran.

\section{La especificación de procesos}

En la especificación que se presenta a continuación, están definidas una serie de recomendaciones que las IES pueden incorporar en sus procesos fundamentales. La funcionalidad implícita (subprocesos) en cada uno de los procesos, el orden lógico (flujo de trabajo) en que estas funciones son realizadas, así como las actividades y acciones que comprende, conforman un marco de referencia conceptual que las IES pueden difundir entre todos los involucrados en sus procesos (trabajadores del negocio), para propiciar el entendimiento común y extender esta percepción hasta los sistemas de información (TI) que soportan el negocio.

\section{Docencia}

El proceso de docencia comprende una de las funciones esenciales de toda IES, está conformado por los subprocesos de: Enseñanza, Tutoría académica y Educación continua (véase fig. I).

Enseñanza. Comprende todas las actividades docentes relacionadas con la impartición de los cursos regulares correspondientes a los programas académicos de la IES. Engloba la preparación de clases, la cátedra, la aplicación de exámenes (evaluación), el desarrollo de prácticas, y la realización de diversas actividades de aprendizaje (pláticas, conferencias, investigación documental, etcétera).

Tutoría académica. Consiste en las actividades encaminadas a fortalecer y consolidar los conocimientos adquiridos en los cursos regulares, a través de una relación directa alumno-profesor. Comprenden las asesorías en temas asociados a los cursos regulares, así como la asistencia en los trabajos de titulación (en las distintas modalidades que ofrezca la IES).

Educación continua. Este subproceso comprende la oferta continua de cursos de actualización y capacitación en temáticas correspondientes al perfil (áreas de conocimiento y especialización) de la IES. Estos estudios no conllevan como objetivo la obtención de un título profesional. 


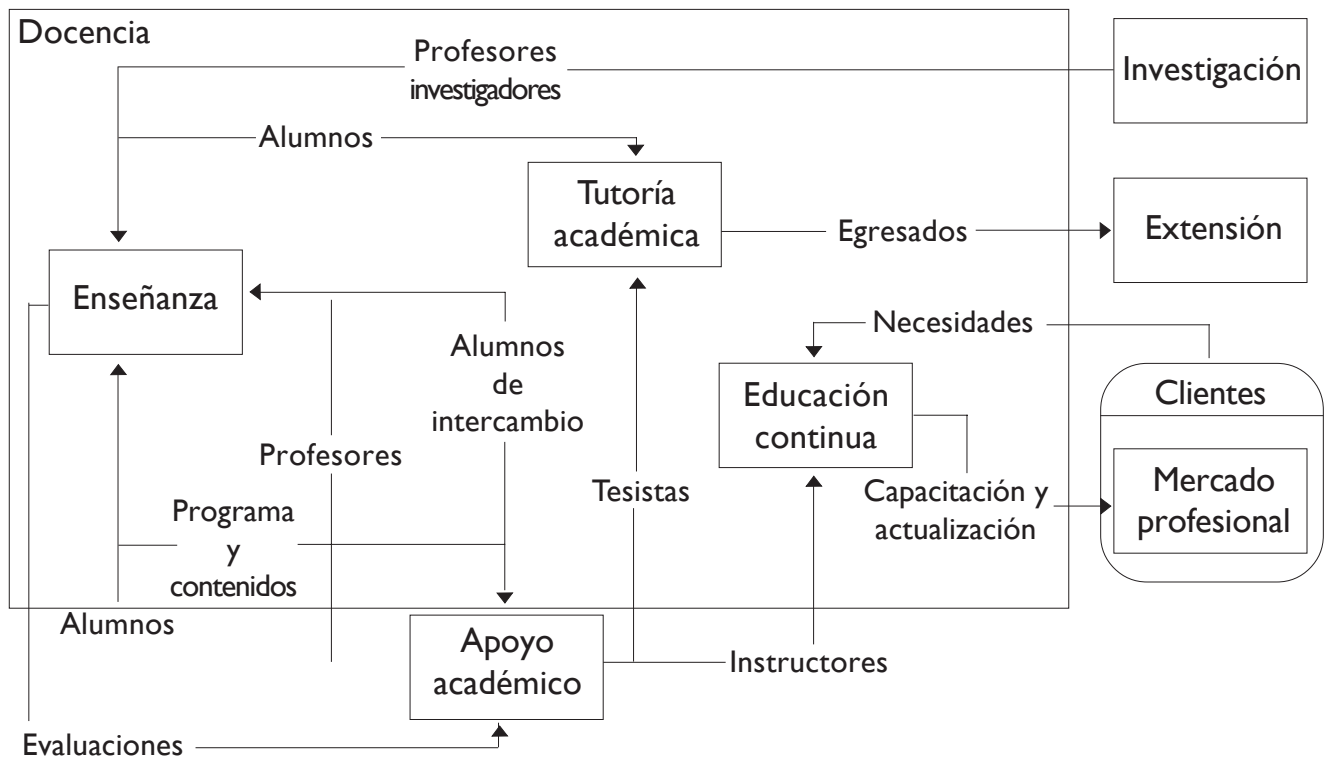

Figura I.Proceso de Docencia en UnA IES

\section{Investigación}

La generación y aplicación del conocimiento es una de las funciones sustantivas en toda IES. Comprende todas las actividades asociadas con el trabajo desarrollado por los investigadores agrupados en organismos colegiados de investigación, los cuerpos académicos. En esta función se fortalece el proceso de docencia y se generan los productos y servicios (conocimiento, asesorías, consultorías, etc.), que se entregan a los clientes de la IES (empresas, comunidad, etc.) a través de las funciones de extensión. El proceso de Investigación está conformado por los subprocesos de:Administración de cuerpos académicos y Desarrollo de proyectos (véase fig. 2).

Administración de cuerpos académicos. Comprende la definición de la Línea General de Aplicación del Conocimiento (LGAC), la definición de las temáticas asociadas a ésta, el desarrollo y consolidación de los cuerpos académicos, la formación y actualización de investigadores, la participación 


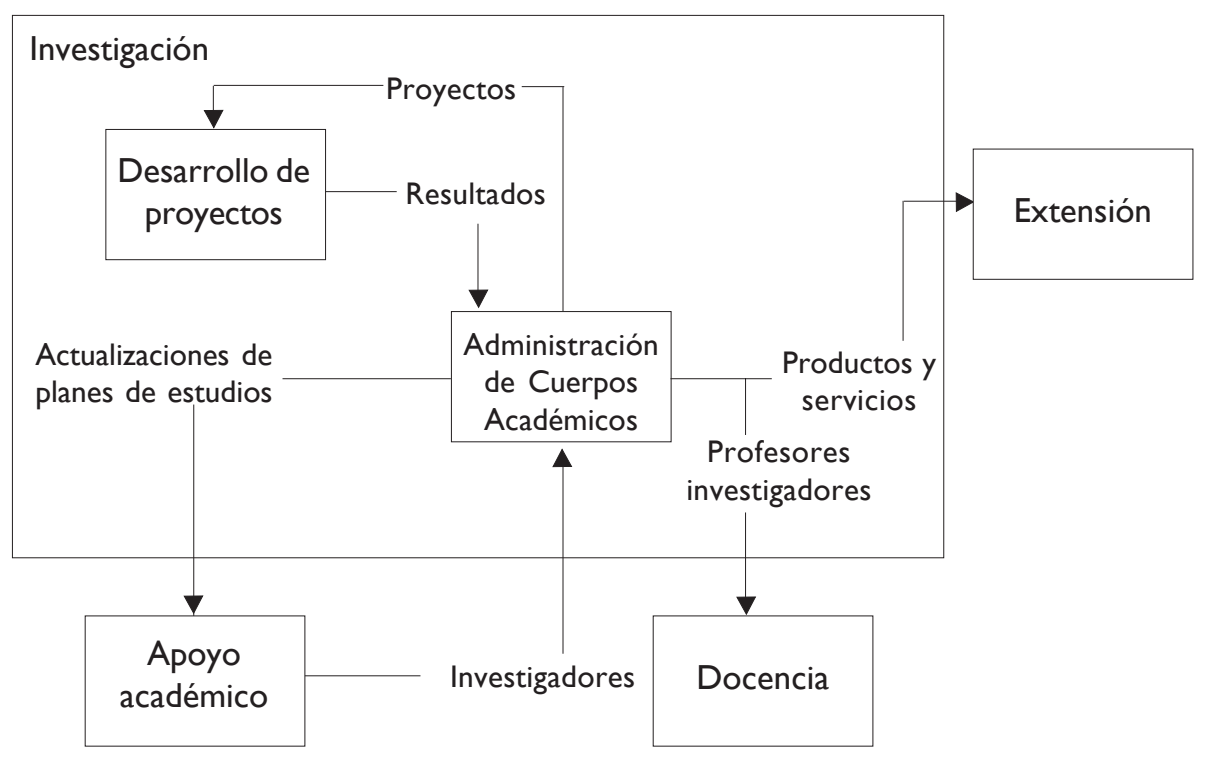

Figura 2. Proceso de INVESTigación EN UNA IES

en proyectos e iniciativas en colaboración con otros cuerpos académicos, la vinculación de las actividades de investigación con las de docencia (la formación de profesores-investigadores y la actualización de los programas académicos), y las de extensión (difusión de productos de la investigación).

Desarrollo de proyectos. Este subproceso engloba el control y seguimiento de las actividades relacionadas con la ejecución de proyectos de investigación, desde la definición de protocolos, pasando por la asignación de recursos humanos, y financieros, hasta la generación de resultados específicos a una problemática (dentro de la LGAC) planteada interna o externamente.

\section{Extensión}

A través del proceso de Extensión la IES entrega a sus clientes (empresas, comunidad, mercado profesional, etc.) los productos y servicios que se generan 
en los procesos sustantivos de Docencia e Investigación. Mediante la Extensión se cumple con el propósito de vincular estos procesos con los requerimientos que se plantean desde el entorno de la IES; está conformada por los subprocesos de: Difusión y divulgación, Centro Emprendedor de Negocios, Seguimiento de egresados, Bolsa de trabajo y Servicio social (véase fig. 3).

Difusión y divulgación. Comprende actividades encaminadas a difundir, a través de diversos medios de información, los productos y servicios resultantes de la función sustantiva de Investigación. Tiene un impacto directo con la comunidad a la que la IES sirve.

Centro Emprendedor de Negocios (CEN). Aquí se comercializan a través de asesorías, consultorías y servicios especializados, los productos y servicios generados por los investigadores de la IES. EI CEN es una empresa parauniversitaria que atiende necesidades de las empresas del entorno, relacionadas con las LGAC de sus cuerpos académicos.

Seguimiento de egresados: Actividades que tienen por objetivo el control y seguimiento del producto principal de la función docencia, los egresados de programas académicos de la IES. Es a través de este proceso como se obtiene información del entorno empresarial de la IES, relacionado con el status de sus egresados que desempeñan su actividad profesional en ellas.

Bolsa de trabajo. Actividad que tiene como único objetivo el registro de los egresados de los programas académicos y su ubicación en las empresas del entorno de la IES.

Servicio social. Comprende una serie de actividades que forman al alumno en el compromiso con la sociedad y proyectan su acción en beneficio de ésta. Comprende actividades profesionales no escolarizadas, que forman parte del plan de estudios sólo que con finalidades de práctica y servicio.

\section{Apoyo académico}

Proceso adjetivo que se desempeña en una IES, el cual agrega las actividades relacionadas con el aseguramiento de la calidad de la función docencia, 


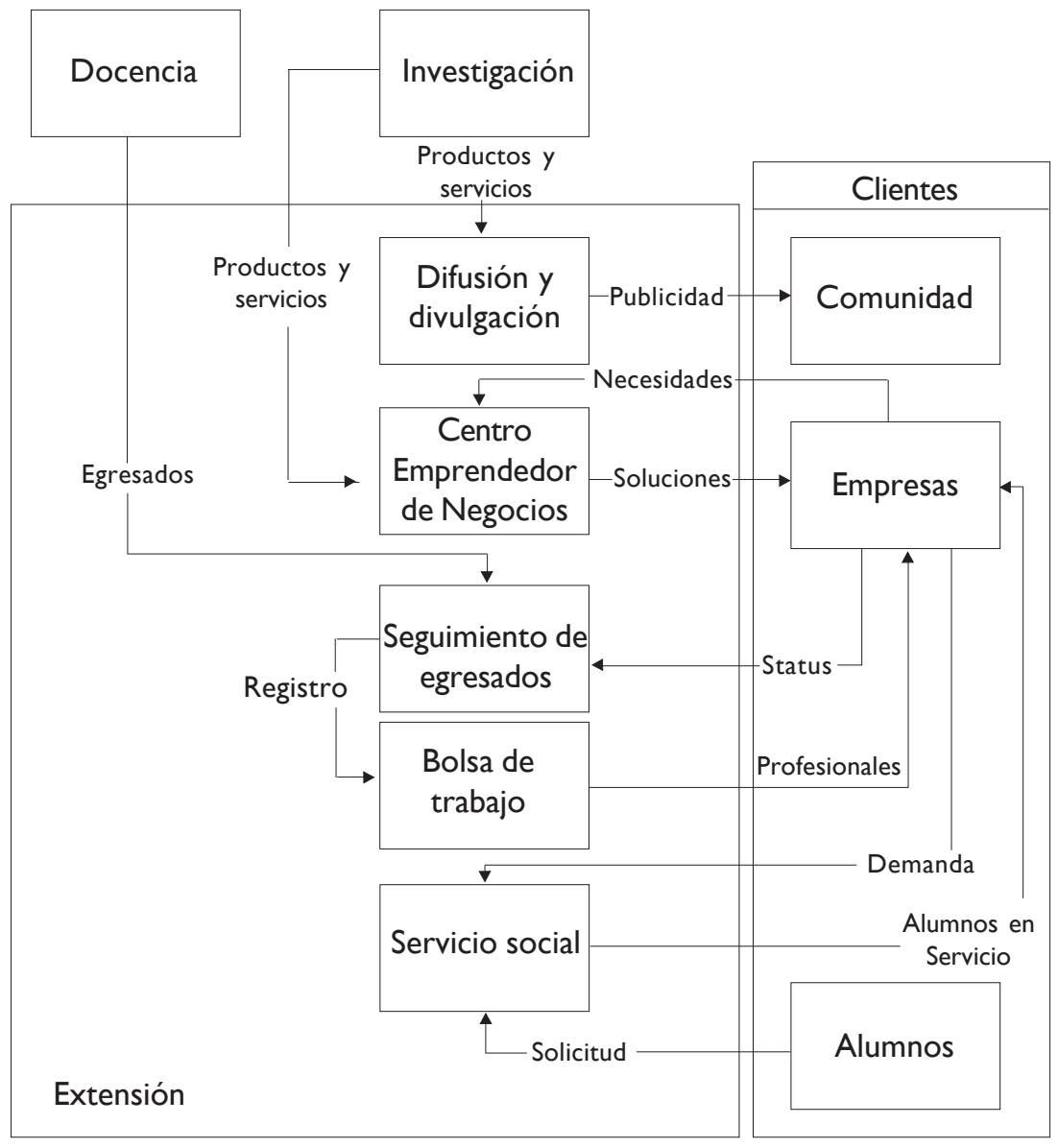

Figura 3. Proceso de Extensión de UnA IeS

investigación y extensión. Este proceso está a cargo de la logística interna que provee de insumos al proceso de Docencia (aspirantes), así como de la atención de solicitudes de diversos servicios que los alumnos (clientes) solicitan. El proceso de Apoyo académico está conformado por los subprocesos de: Admisión, Inscripción, Programación académica, Administración 
La especificación de

procesos en las IES

de planes de estudio, Administración de currícula, Intercambio académico, Administración de servicios escolares, Bienestar estudiantil, Titulación y Registro escolar (véase fig. 4).

Admisión. Subproceso que comprende las actividades relacionadas con el ingreso de nuevos estudiantes a las IES, desde que solicitan información de los programas académicos hasta que presentan su examen de nuevo ingreso, entregan documentación y se les asigna una clave de identificación (matrícula).

Inscripción. Subproceso que comprende el registro de los alumnos en los cursos que las divisiones académicas de las IES impartirán durante un periodo escolar.

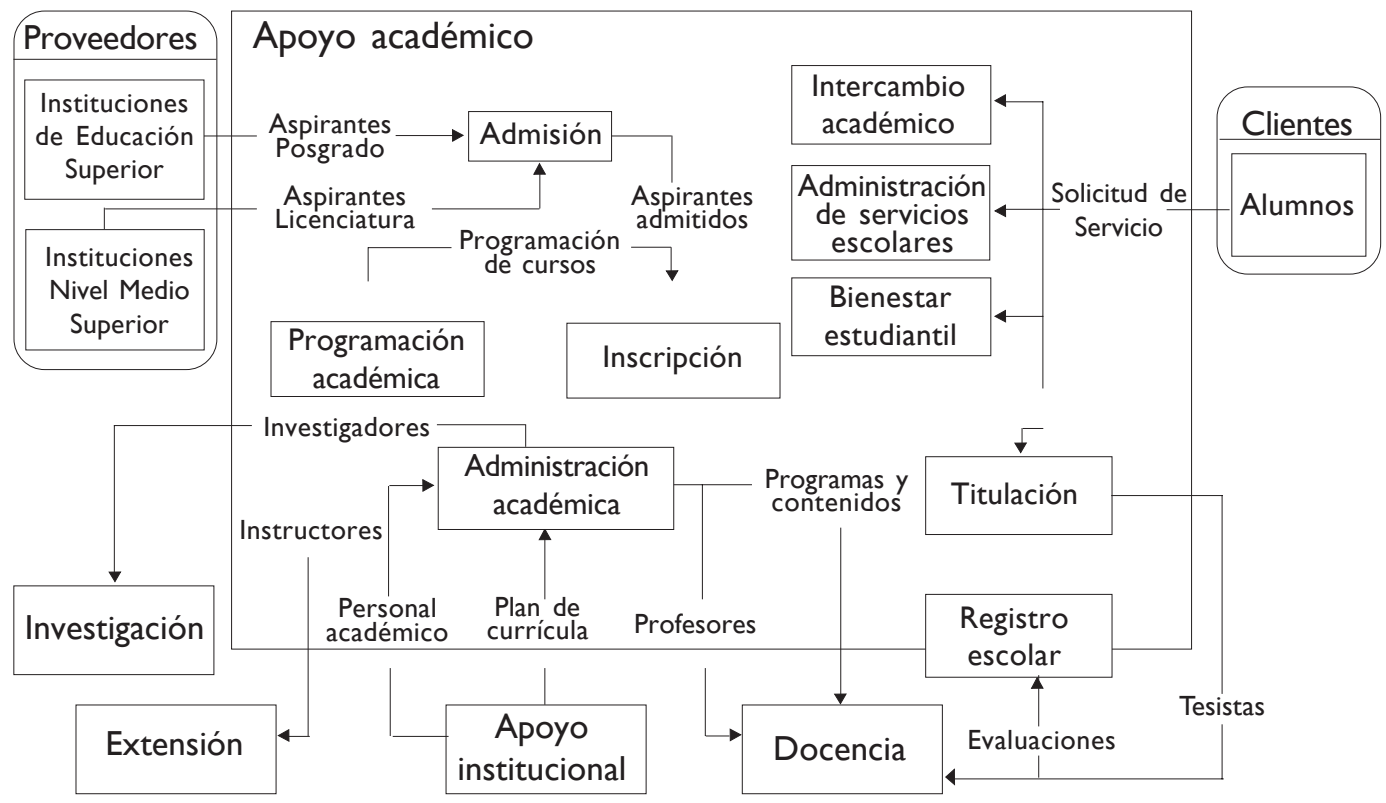

Figura 4. Proceso de Apoyo académico de una ies 
Programación académica. Actividades encaminadas a definir la oferta de cursos regulares y exámenes especiales (extraordinarios) correspondientes a un periodo escolar.

Administración académica. Subproceso que incluye a todas las actividades relacionadas con la planeación, creación y actualización de planes y programas de estudio. Comprende la definición y actualización de objetivos, materias/cursos, contenidos, actividades de aprendizaje, perfil del aspirante, perfil del egresado, mapa curricular, tiempos y formas de evaluación. Asimismo, comprende a las actividades enfocadas en la definición de los perfiles de profesores e investigadores que requieren los procesos de docencia e investigación, la evaluación de nuevos profesores (concurso de oposición), curso de inducción al personal académico, capacitación en habilidades didácticas, tutoriales. Incluye todas las actividades encaminadas a generar habilidades para la enseñanza.

Intercambio académico. Incluye todas las actividades realizadas para fomentar la movilidad estudiantil y docente que incrementa los vínculos de cooperación con otras IES, nacionales y extranjeras.

Administración de servicios escolares. Actividades encaminadas al control y seguimiento académico de los alumnos, y al cumplimiento de normas y reglamentos académicos (reglas del negocio). Comprende el cambio de carrera, emisión de documentos oficiales, cambios de situación académica (bajas temporales y definitivas), revalidaciones, etcétera.

Bienestar estudiantil. Actividades cuyo propósito principal es el de contribuir al bienestar emocional y físico del estudiante, así como a su desarrollo intelectual, cultural y social fuera del contexto del programa educativo.

Titulación.Actividades encaminadas a la certificación de los estudios a través de diversas modalidades. Comprende la revisión de estudios, la selección de tema de tesis, la elección del director de tesis, la designación de revisores, la designación de sinodales, la emisión de documentos oficiales (actas), el registro del resultado del examen profesional (o de grado), el registro ante la Dirección General de Profesiones, el trámite del título. 
Registro Escolar: Subproceso que comprende el mantenimiento al día, de las calificaciones y seguimiento académico de los estudiantes.

\section{Apoyo Institucional}

Proceso adjetivo de las IES, que agrega las actividades relacionadas con el gobierno, la planeación, la administración y el soporte de la institución, así como la disposición permanente y oportuna de los recursos humanos, materiales y financieros necesarios para la operación de la IES. El proceso de Apoyo institucional comprende los subprocesos de: Gobierno, Planeación, Administración, Finanzas, Recursos humanos, Recursos materiales y Servicios de cómputo (véase fig. 5).

Gobierno. Actividades de la dirección / rectoría en la IES, enfocadas en el planteamiento de estrategias institucionales, la dirección y el control desde una perspectiva integral. Este proceso tiene entre sus principales productos las líneas estratégicas que conforman el Plan Estratégico de Desarrollo Institucional (Plades) y las del Programa Integral de Fortalecimiento Institucional (PIFI).

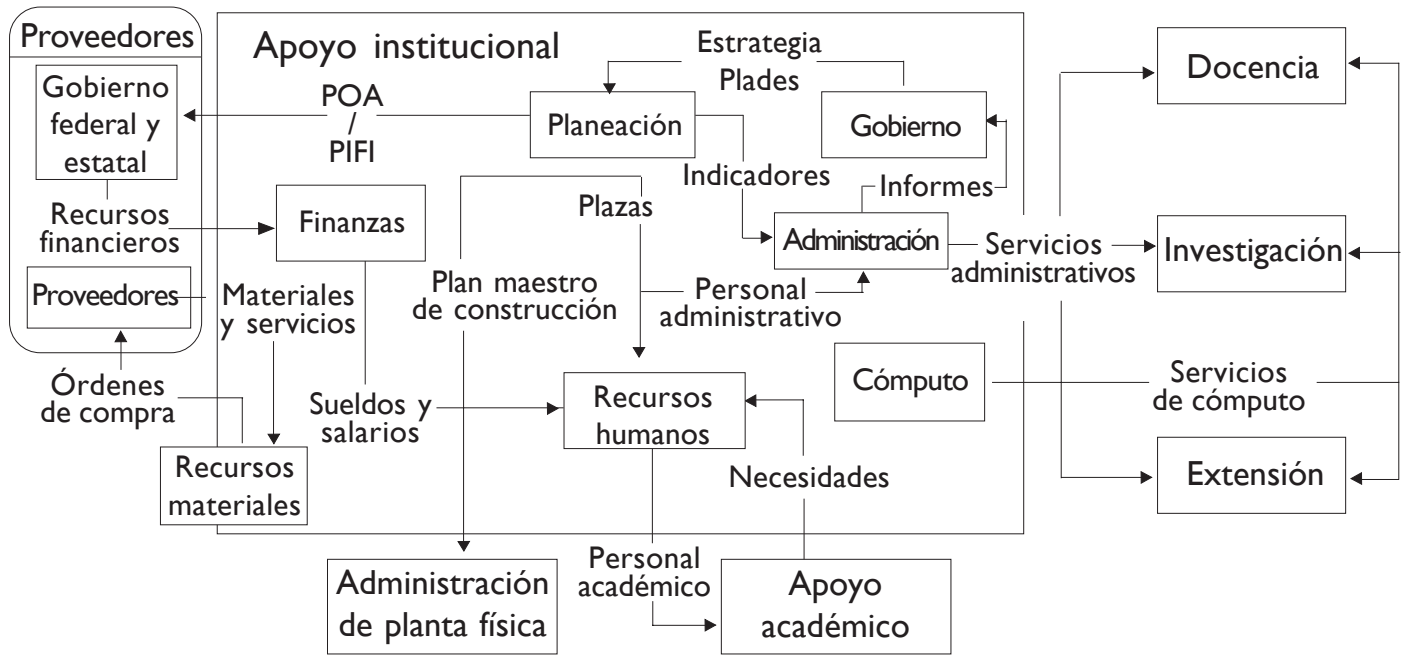

Figura. 5. Proceso deApoyo institucional de UnA ies 
Planeación. Este subproceso comprende las actividades encaminadas a definir las acciones que promueven el desarrollo de las funciones sustantivas y adjetivas de la IES. Incluye la elaboración de diagnósticos, el planteamiento de metas y acciones asociadas con las líneas estratégicas de la institución (Plades, PIFI), la previsión y asignación de recursos a través del Programa Operativo Anual (POA) y la evaluación institucional.

Administración.Agrega las actividades de la administración central de la IES, orientadas a asegurar el suministro de recursos humanos, materiales y financieros necesarios para la operación del sistema. Incluye las actividades de las unidades de apoyo administrativo de la institución, tendientes a la organización y control.

Finanzas. Subproceso que agrega las actividades destinadas a la administración de los recursos económicos, así como al tratamiento de la información resultante del manejo de los mismos.

Recursos humanos. Subproceso que incluye las actividades encaminadas a la administración del personal, tanto académico como administrativo.

Recursos materiales. Este subproceso incluye las actividades asociadas con la administración de los servicios complementarios de apoyo, para llevar a cabo la operación normal de la institución, tales como adquisiciones, control de inventarios, control patrimonial, servicio de correo, mensajería, transportación e infraestructura de comunicación.

Servicios de cómputo. Comprende las actividades asociadas con el suministro de servicio de cómputo a las áreas académico-administrativas y administrativas de la institución, tales como el diseño de sistemas de información, bases de datos y sistemas de soporte a las decisiones.

\section{Administración de planta fisica}

Proceso adjetivo de las IES que agrega las actividades relacionadas con la administración, operación, conservación, mantenimiento, expansión y ampliación de la planta física. El proceso de Administración de planta física comprende los subprocesos de:Administración de infraestructura, mantenimiento y conservación, Expansión y modificación (véase fig. 6). 
Administración de infraestructura. Agrupa las actividades relacionadas con la administración y operación de la planta física, el cuidado y la atención de los bienes muebles e inmuebles

Mantenimiento y Conservación. Comprende las actividades de mantenimiento preventivo y correctivo de la planta física, así como el mantenimiento de inmuebles, jardinería y campos.

Expansión y Modificación. Actividades encaminadas a la modificación, ampliación, y adaptación de la planta física, así como a la construcción de nuevas instalaciones.

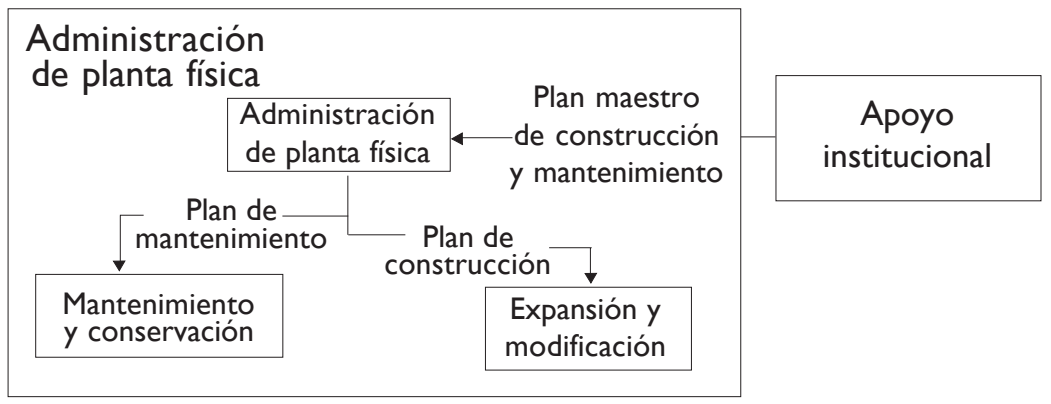

Figura 6. Proceso de Administración de planta física de UNA IES

\section{Conclusiones}

La especificación de procesos presentada conforma un marco de referencia desde una perspectiva sistémica de los procesos "core" que se realizan en toda IES. Esta especificación está fundamentada totalmente en la arquitectura de procesos de la institución y comprende una serie de recomendaciones que las IES pueden asumir para reordenar las actividades y tareas que en ellas se llevan a cabo.

La funcionalidad implícita en cada uno de los procesos/subprocesos, el orden lógico en que estas funciones son realizadas, así como las actividades y acciones que comprende, conforman un marco de referencia conceptual para las IES, el cual debe ser difundido entre los responsables de desempeñar 
las actividades de valor y las unidades organizacionales donde se llevan acabo, con la finalidad de propiciar el entendimiento de la empresa y permear esta percepción hasta los sistemas de información que soportan el negocio.

\section{FUENTES CONSULTADAS}

Maldonado T., Armando (2003). Arquitectura de procesos. Curso de arquitectura de la empresa. México: ITAM.

Porter, Michael E. (2002). La ventaja competitiva, ed. rev. México: CECSA.

Rummler, Geary A. y Alan P. Brache (1990). Improving Performance: How to Manage the White Space on the Organization Chart. San Francisco: Jossey-Bass.

Velázquez, Adalberto y Armando Maldonado (2005). "Una arquitectura de procesos para las instituciones públicas de educación superior". Teoría y Praxis, I, (I), Cozumel: Universidad de Quintana Roo. 
